Archives

$44 \mid 2009$

Varia

\title{
L'histoire tragique et mémorable de Claude de Sainctes évêque d'Évreux
}

\section{Marco Penzi}

\section{(2) OpenEdition}

\section{Journals}

Édition électronique

URL : http://journals.openedition.org/ccrh/3522

DOI : $10.4000 /$ ccrh.3522

ISSN : 1760-7906

Éditeur

Centre de recherches historiques - EHESS

Édition imprimée

Date de publication : 25 janvier 2009

Pagination : 9-26

ISSN : 0990-9141

\section{Référence électronique}

Marco Penzi, "L'histoire tragique et mémorable de Claude de Sainctes évêque d'Évreux », Les Cahiers du Centre de Recherches Historiques [En ligne], 44 | 2009, mis en ligne le 25 novembre 2011, consulté le 19 avril 2019. URL : http://journals.openedition.org/ccrh/3522 ; DOI : 10.4000/ccrh.3522

Ce document a été généré automatiquement le 19 avril 2019

Article L.111-1 du Code de la propriété intellectuelle. 


\title{
L'histoire tragique et mémorable de Claude de Sainctes évêque d'Évreux
}

\author{
Marco Penzi
}

1 L'histoire de la Ligue, telle qu'on la connaît généralement, est racontée à travers les grands événements emblématiques de la période 1585-1594: on peut en effet la reconstituer à travers la «Journée des barricades» en mai 1588, le siège de 1590, l'assassinat d'Henri III en 1589, celui du premier président Brisson en 1591, le siège de Rouen, la conversion du roi Henri IV en 1593, ou encore l'entrée dans Paris en mars 1594.

2 Par contraste, les historiens de l'époque marquent un goût particulier pour la narration des péripéties au quotidien : Jacques Auguste de Thou, Palma Cayet et Pierre Matthieu rappellent sans cesse la réalité de cette guerre : sièges faits et villes enlevées, batailles qui ne sont que des escarmouches, difficultés infinies dans les armées : approvisionnements, soldes des soldats, manque d'armes ou de poudre, incompétence des belligérants. Le tout accompagné du nom des auteurs des «faits mémorables », bons ou mauvais. L'histoire de cette huitième et dernière guerre de Religion semblerait donc se réduire aux « prises » de telle ou telle ville, presque toujours par la ruse ou la trahison.

3 Cet aspect du récit historique que l'on trouve chez les auteurs de l'époque est confirmé par la «presse » ligueuse : il suffit de jeter un œil rapide sur le Catalogue établi par Denis Pallier pour prendre connaissance des trahisons des politiques qui ont permis aux soldats de Henri de Navarre de s'emparer d'une ville ou bien d'un village fortifiéi ${ }^{1}$. De la sorte, à côté des "trahisons", on retrouve, dans les récits ligueurs et royalistes, les « compositions » négociées, les accords pour la reddition d'une ville, concernant ce qu'on appelait hier l'honneur des armes. À l'époque, garder « armes et bagues sauves » était un fait plutôt banal qui ne comportait pas toujours une reconnaissance de l'honneur des assiégés.

4 Ainsi Évreux, le siège épiscopal où siégeait le théologien Claude de Sainctes*, fut prise par composition par Biron le 20 janvier 1591. L'évêque, qui avait signé le serment de la Ligue, fut persuadé de s'enfuir et se retira à Louviers; ce fait, qui est passé presque inaperçu 
dans les livres d'histoire, étant donné le peu d'importance qu'on lui prête face à l'Histoire fut le début des événements que j'essaierai d'élucider.

\section{Un contexte historique et historiographique}

5 L'année 1591, est probablement une des moins connues de l'histoire de la Ligue : mis à part l'assassinat de Brisson, et le début du siège de Rouen, il reste peu de choses dans la mémoire collective, si on peut parler ainsi des événements de cette année-là. Pourtant 1591 fut l'année de l'expédition de l'armée pontificale, et aussi l'année, la seule d'ailleurs, du pontificat de Grégoire XIV. 1591 marqua surtout un temps fort dans le conflit entre les parlements royalistes de Tours et de Châlons et la papauté; des événements que l'on peutà juste titre qualifier de "crise gallicane ", une crise encore plus forte que celle de 1551. Je vais donc m'intéresser à un événement particulier pour démontrer comme tout est loin d'être dit sur l'histoire de la Ligue : la façon dont elle nous a été narrée est le récit des événements établi par les historiens royalistes; parfois, la réalité des choses est cachée sous des demi-mensonges dictés par les appartenances politiques du moment. Cette affirmation est suggérée par une constatation simple : aucun des historiens qui ont conté la « guerre de la Ligue » n'a produit un récit que l'on pourrait qualifier de vision ligueuse de l'histoire. Dans les textes de Jacques Auguste de Thou, Palma Cayet, Pierre Matthieu, Jean-Baptiste Legrain et bien sûr Pierre de l'Estoile, pour ne citer que les plus connus, on ne trouvera aucune "véritable » justification de la Ligue, aucune marque de sympathie ou de compréhension à l'égard d'un mouvement constamment qualifié, du moins après coup, de rébellion contre l'autorité royale légitime, celle de Henri IV. Ce fait s'explique par deux raisons : ces écrivains étaient membres du parti des « Politiques » ou bien ils détenaient une charge d'historiographe qui leur avait été confiée par la royauté ${ }^{2}$. Cette considération, qui a été relativement oubliée par l'«Histoire» des guerres de Religion et donc par les historiens, était en revanche bien présente à l'esprit des contemporains, en particulier ceux qui avaient vécu l'expérience de la guerre civile de l'autre côté des barricades, celles justement de la Sainte Union.

6 L'ancien ambassadeur savoyard René de Lucinge en 1614 dans son livre La manière de lire l'histoire critiquait durement Cayet et Matthieu. Lucinge les accusait d'avoir menti au sujet des événements historiques, d'avoir voulu expressément cacher au lecteur certains aspects pour fournir une interprétation altérée qui fut le plus favorable possible au parti de Henri IV . De la même façon, au long de son récit, l'auteur anonyme de L'histoire de la Ligue, n'hésite pas à rappeler les événements en soulignant à plusieurs reprises, quand le cas semble digne d'attention, qu'une version différente peut être trouvée chez un historien non royaliste ou dans des libelles contemporains ${ }^{4}$.

7 L'historiographie adhérant inconsciemment ou consciemment au point de vue des Politiques, à cette histoire façonnée à l'usage du parti royal vainqueur de la guerre civile, a déversé et déverse encore aujourd'hui sur la Ligue les mauvaises appréciations que ses adversaires, les Politiques, ont confiées à la mémoire. Il est bien évident, que si les Politiques se définissaient, dans leurs écrits et histoires, comme le parti national et les défenseurs de la royauté et de la monarchie, les ligueurs ne pouvaient rien être d'autre que des traîtres, des serfs de l'or espagnol, des violents et des fanatiques. Ainsi une histoire destinée à transmettre pour l'éternité cette version partisane ne pouvait que, sinon effacer totalement, du moins cacher ou rendre moins évidentes les faiblesses politiques ou les hésitations religieuses dont les Politiques avaient fait preuve durant ces 
années de lutte. Une fois la paix revenue dans le royaume, après qu'Henri IV se fut converti à l'Église catholique, mais surtout se fut rapproché de Rome et de la papauté, la «nouvelle » histoire des Politiques, qui était en même temps l'histoire de la «nouvelle» monarchie, ne pouvait pas relater des événements " politically incorrect ».

Les historiens d'Henri IV représentent de cette manière tout acte des partisans du roi seulement comme une réponse à une agression du parti opposé et jamais comme une action volontaire ou faisant référence à une conception politique précise. Par exemple, l'action schismatique entreprise par les parlements royalistes entre le printemps et l'été 1591, et entretenue chez certains esprits au moins jusqu'en 1593, est racontée par les historiens du premier Bourbon en passant sous silence tout acte extrême que leur parti avait accompli contre les ligueurs, qu'ils fussent laïcs ou ecclésiastiques.

\section{Claude de Saintes, évêque (1524-1591)}

9 Le sujet que j'entends développer dans les pages suivantes - l'histoire de Claude de Saintes - est, dans ce sens, une anti-histoire. Sur ce personnage, on dispose d'une série de "sources", qui malheureusement se recopient l'une l'autre sans rien ajouter à la triste fin de cet ecclésiastique. Claude de Saintes, évêque d'Évreux, né en 1524 dans le Perche au diocèse de Chartres ( $d$ 'ancienne famille noble, selon l'abbé Chemin, roturier selon Baumgartner) $)^{5}$, entra en 1534 chez les chanoines réguliers de Saint-Chéron-les-Chartres ; il se fit religieux en 1540. Il poursuivit ses études au collège de Navarre et fut ensuite pourvu de la cure de Bainville-le-Comte. Après avoir été élu principal du collège de Boissy, grâce au Cardinal de Pellevé, archevêque de Sens, et à Dusouchier abbé de Clairvaux, il fut présenté au cardinal de Lorraine, qui remarqua ses qualités et le prit dans sa maison ${ }^{6}$.

10 En 1560-1561, il publia un écrit pour montrer que les princes ne doivent point tolérer les hérétiques ${ }^{7}$. Ses talents lui permirent de participer au colloque de Poissy de 1561. Relativement à cette assemblée il publia une Confession de la foy catholique contenant en brief la reformation de celle que les ministres de Calvin presentèrent au roy en l'assemblée de Poissy, adressée au peuple de France ${ }^{8}$, texte qui rappelait le devoir du catholique d'avoir de la haine pour toute hérésie. L'année suivante il fera partie de la délégation française au concile de Trente ${ }^{9}$. En 1575, après d'autres travaux, il fit paraître son fameux traité sur l'Eucharistie, alors que, sur la fin de 1574, il avait été nommé par Charles IX évêque d'Évreux (il fut confirmé par Henri III). En 1581, il travailla à la rédaction en français des actes du concile provincial tenu à Rouen. Quelques années plus tard, en 1586, il fournit à ses curés un nouveau bréviaire, et, en 1587, lors d'un autre synode, il proposa la réception des décrets du concile de Trente. À son sujet, on rappelle que son anti-calvinisme était si fort qu'il soutenait qu'il fallait rebaptiser les calvinistes qui se convertissaient ${ }^{10}$.

11 Présent aux États de Blois en 1588, il s'en retira après l'assassinat des Guise et, après un bref passage par Paris, où il « signa la Ligue », il s'installa définitivement dans son évêché d'Évreux. Les meurtres commis sur Messieurs de Guise lui parurent des crimes irrémissibles. Arrivé à Évreux, il s'en plaignit avec amertume. Fortifié ensuite comme tout le monde par le décret de la Sorbonne qui,

décidoit que les François estoient desliées (sic) du serment de fidelité preté au Roy, qu'on pouvoit en conscience prendre les armes, former une ligue, lever de l'argent, et recourir à tous les moyens nécessaires pour la conservation de la religion catholique contre les mauvais desseins dudit seigneur Roy, déclarant tous les 
moyens de deffense légitimes depuis qu'au preiudice de la religion catholique et de l'édit de lunion (sic), il avoit violé les lois de la liberté naturelle par les meurtres qu'il avoit commis à Blois, il ne lui fut pas difficile de déterminer Évreux à tenir teste au souverain légitime ${ }^{11}$.

Plus que jamais ligueur, de Saintes devient même l'âme de la Ligue à Évreux. Quand, en 1591 , la ville tomba aux mains des royalistes, de Saintes se réfugia à Louviers. Cette ville, le 6 juin de la même année, fut prise à son tour. Cette fois, de Saintes fut fait prisonnier.

\section{L'histoire tragique et mémorable de l'évêque d'Évreux}

13 Le reste de l'histoire de notre évêque, on la laissera raconter par les historiens et mémorialistes de l'époque : Pierre de l'Estoile narre brièvement la prise de Louviers. Au sujet de l'évêque, il écrit sans rien ajouter de plus ${ }^{12}$ :

le roi l'a mis entre les mains du parlement de Caen, pour avoir fait quelques écrits où il prétend justifier le parricide commis sur Henri III, et prouver qu'il est permis d'en faire le même sur le roi de Navarre.

Pierre Matthieu n'est pas plus clair :

le Roy ayant dés le mois de septembre 1591 surpris Louviers, chastié quelques seditieux, et envoyé prisonnier à Caen l'Evesque d'Évreux, nommé de Sainctes, l'un des arcs boutans de la Ligue $[. . .]^{13}$

Palma Cayet raconte avec beaucoup de soin la prise de Louviers grâce à la «trahison » de certains habitants, dont un prêtre, mais au sujet de Claude de Saintes, il nous dit seulement qu'il fut mené prisonnier à Caen ou il mourut peu de jours après ${ }^{14}$.

De ces récits deux choses sont à retenir :

- de Saintes, "arc-boutant» de la Ligue, est l'auteur d'écrits tyrannicides contre les personnes de Henri III et Henri IV ;

- il a été envoyé prisonnier au Parlement de Caen ${ }^{15}$.

19 De Thou, heureusement, nous permet de savoir comment se termina cette « histoire » que les autres ont laissée en suspens. Le sens du récit de Jacques A. de Thou est donné par le titre en marge de son récit: «Le fanatique Claude de Sainte [... ] est pris $»^{16}$ : comme Cayet, il rappelle les circonstances de la prise de la ville; il évalue à vingt le nombre des prisonniers dont,

l'évêque d'Évreux, fameux théologien, zélé ligueur, et l'un des plus obstinés ennemis du Roi. On s'empara de ses livres; et on y trouva parmi ses papiers un écrit où il justifioit l'assassinat d'Henri III et s'efforçoit de prouver qu'il était permis de tuer le roi de Navarre : c'est pourquoi on ne le traita pas comme un prisonnier de guerre ; mais on l'envoya à Caën sous bonne garde, pour lui faire son procès, et le punir comme un criminel de lèze-Majesté : car on n'a point d'égard en France aux prérogatives du Clergé, lorsqu'il s'agit d'un crime d'Etat; et la sévérité des lois tombe indifféremment en ce cas sur les Evêques et sur les Prêtres, comme sur les laïcs. Il ne s'en fallut rien que ce séditieux prélat ne fut condamné à mort. Il fut atteint et convaincu. Il osa même défendre opiniatrement les malheureuses et extravagantes opinions dont il estoit entêté. Le Cardinal de Bourbon et le Clergé du parti du Roi intercédérent pour lui : on obtint que la peine de mort, qu'il avoit encourue selon nos loix, seroit commuée en prison perpetuelle, où il mourut peu de temps après ${ }^{17}$.

Voilà donc l'explication de l'historien : Claude de Saintes, criminel de lèse-majesté (car auteur d'écrits tyrannicides) est condamné à mort. Jacques de Thou nous explique aussi 
que les lois françaises ne prévoient pas d'exemption pour les ecclésiastiques coupables de crimes pareils. La justice gallicane, nous dit l'auteur, ne remet pas les accusés ecclésiastiques entre les mains des tribunaux du clergé. Enfin l'intercession du cardinal de Bourbon auprès d'Henri IV et la bonne volonté du roi (de Thou ne le dit pas, mais c'est implicite dans son récit) firent que de Saintes sera condamné à perpétuité au lieu de mourir écartelé ou mis à mort d'autre façon, comme c'était les cas pour les accusés de lèse-majesté.

21 Nous pouvons donc conclure que l'histoire de Claude de Saintes se réduit pour nos historiens à un crime de lèse-majesté puni par la justice selon les lois françaises, condamnation commuée en prison perpétuelle par un acte noble du souverain. Bayle commente ainsi ce « beau geste » :

Henri IV agit sans doute dans cette occasion par les principes de la clémence et de la générosité qui lui étaient naturelles; mais il s'y mêla aussi un peu de cette prudence timide qui ébranla si souvent son grand courage, après qu'il eut observé que le monstre de la ligue qu'il avait à vaincre, plus farouche et plus dangereux que l'hydre d'Hercule, deviendrait et plus furieux et plus indomptable par l'effusion de son sang. C'est pourquoi ce prince se crut obligé à se servir de la douceur afin d'apaiser et d'apprivoiser cette bête si féroce. La clémence d'un côté, et la politique de l'autre, épargnèrent à Claude de Sainctes la honte de perdre la vie sur un échafaud, comme il l'avait mérité 18 .

Il faut de nouveau souligner, que de Thou parmi ses contemporains est le seul à éclaircir les faits. On dirait presque le crime "classique » ou "emblématique » d'un prédicateur ligueur. De Thou trouve quasi-naturel que les crimes d'État soient punis, et ne fournit pas de longues explications pour défendre l'acte et la théorie qui fondent la sentence du Parlement. Le jugement est donné, personne n'a songé à justifier en quelque sorte l'évêque, sa condamnation est en un certain sens préalable au procès; selon ces historiens, son crime est simplement inexcusable et on n'en parlera plus.

\section{Autour de la condamnation d'un évêque : procès et contexte}

23 Mais un évêque condamné à mort n'est pas un cas judiciaire quelconque. Jules Lair, dans son étude sur le parlement normand, nous fournit quelques précisions : selon son récit, le Parlement averti de l'emprisonnement de l'évêque de Saintes envoya le conseiller Godefroid pour s'emparer de lui. C'est au moment de l'arrivée de cet émissaire du Parlement qu'on fouilla dans les papiers de l'évêque et qu'on y trouva la pièce qui l'accusait ${ }^{19}$. Deux questions attirent notre attention:

24 - les écrits de l'évêque étaient-ils des papiers, un livre imprimé, des manuscrits ou bien un ouvrage de ce dernier sur le tyrannicide? On ne le saura probablement jamais ;

25 - le Parlement de Caen voulait l'emprisonner avant la découverte des papiers compromettants; quel était donc le crime de l'évêque de Saintes ? D'ailleurs, pourquoi fouiller dans les affaires de l'évêque si l'on n'avait pas de soupçons sur lui?

Amable Floquet dans son Histoire du Parlement de Normandie, consacre aussi plusieurs pages à l'histoire de l'évêque d'Évreux. Son jugement n'est pas impartial. Pour Floquet, de Saintes était un séditieux, auteur d'écrits contre le souverain, qui fut justement condamné par le parlement. Mais sa seule source au sujet du procès est le récit de Jacques de Thou que l'on a analysé plus haut. Il faut souligner que, semble-t-il, les actes du procès 
sont perdus, il est donc presque impossible d'en connaître les circonstances et le déroulement. Selon Floquet, de Saintes une fois fait prisonnier avait failli s'échapper en payant les soldats : le parlement ligueur de Rouen avait rendu un arrêt pour lui permettre « d'engager le revenu de son évesché jusqu'à la somme de mille escus, pour le payement de sa rançon en laquelle il avoit esté cottisé par les ennemys qui le tenoient prisonnyer ${ }^{20}$ - Floquet trouve cette résolution $d u$ parlement rouennais scandaleuse, mais, contrairement au jugement de l'historien, cette façon d'agir était naturelle et pratiquée par tous à l'époque. Personne ne se serait scandalisé si l'évêque avait été libéré après avoir payé sa rançon. De tels usages étaient monnaie courante dans les guerres. Floquet rappelle que la requête du Parlement de Caen, pour l'« extradition » de l'évêque datait du 10 juin 1591 :

Sire, puisque vous avez en vos mains les plus factieux personnages et qui ont les plus troublé le repos de vos subjectz et oublié la foy et fidélité par eulx reitérée, oultre plusieurs maulx par eulx faictz, envoyez-les à vostre justice souveraine, afin qu'ils rendent compte de leurs actions, suivant les loix de vostre royaulme [...] dont tous vos subjectz recevront autant de contentement que ce sera de terreur à voz ennemys et rebelles, et à nous espérance certaine de veoir de bref, la fin de noz misères ${ }^{21}$.

On peut tirer deux conclusions de ces relations des faits. Quatre jours seulement après la prise de Louviers, le parlement royaliste demandait de pouvoir juger l'évêque. L'arrêt du parlement ligueur, en revanche, date de septembre. Selon les royalistes, il y avait donc une urgence pour faire un exemple, comme ils le disent eux-mêmes ; selon les ligueurs, la regrettable captivité de l'évêque n'aurait pas mis en danger sa vie au moins pas dans l'immédiat. La découverte des papiers compromettants, si elle est bien réelle, n'est certainement pas la seule cause de la requête du parlement royaliste. Pourquoi donc en voulaient-ils à de Saintes? Si le récit que Jules Lair fait de l'entrevue entre de Saintes et Henri IV est vrai, l'évêque n'avait-il pas été pardonné par son roi ${ }^{22}$ ? Quel crime avait-il commis en juin 1591, puisque, manifestement, on fouilla tard dans ses papiers? D'autres personnages connurent le pardon royal, comme, en 1604, Loys Dorléans qui avait pourtant écrit des choses atroces contre le Béarnais et sa mère, et un évêque aussi fameux que de Saintes aurait été sûrement très " rentable » pour le souverain dans son économie de reconquête du royaume: les catholiques, tous partis confondus, auraient sûrement apprécié qu'un de leurs théologiens les plus célèbres fût protégé dans l'entourage royal, (prisonnier, mais non maltraité), plutôt que de le voir condamné à mort.

Pour comprendre, il faut faire un pas en arrière : 1591, tout est là, tant du point de vue général que dans le cas particulier de Claude de Saintes. L'année s'ouvre avec le pontificat de Grégoire XIV, qui fut élu le 5 décembre 1590. Ce Pape décida d'intervenir militairement en France. Son neveu Hercole Sfrondato fut nommé chef de l'armée pontificale et il arriva effectivement en France, pour participer avec l'armée du duc de Parme à la défense de Rouen, assiégée par les royalistes. Dès le début de l'année, on comprit que la politique pontificale en France allait sensiblement changer de celle de Sixte V. Le pape produisit différents écrits : un premier connu dès le 22 février 1591 promettait de l'argent aux ligueurs $^{23}$; un deuxième envoyé à Mayenne comportait les mêmes promesses ${ }^{24}$; suivent ensuite le bref envoyé à la faculté de Théologie et celui à la ville de Paris ${ }^{25}$. Dans ces lettres, on rappelait le soutien de la papauté aux catholiques, l'envoi d'une armée et d'un nonce pour parvenir à l'élection d'un souverain catholique. Cette campagne de "propagande » catholique romaine se poursuivit par des lettres de l'ancien légat Caetani et des brefs adressés à divers personnages de haut rang. Dans cette propagande la pièce 
(les pièces, plutôt), la plus importante sont les Bulles de N. S. Père le pape Grégoire XIIII [...] ${ }^{26}$. Selon ces bulles, les ecclésiastiques qui suivent le parti de Navarre devaient le quitter, sous peine d'être excommuniés, suspendus ou interdits. La même injonction était adressée à la noblesse et aux laïcs. La stratégie romaine était désormais claire : avec des «foudres" spirituelles, on essayait de détacher de Navarre les ecclésiastiques et les gentilshommes et officiers catholiques. Ces textes prévoyaient des sanctions très dures pour les ecclésiastiques et assez sévères pour les laïcs: on rappelait qu'une excommunication pontificale entraînait la perte de toute fonction, de tout bien personnel et levait toute obligation de fidélité de la part des sujets (ce fut le cas du roi de Navarre en 1585).

Cette démarche romaine, ne pouvait pas passer inaperçue chez les royalistes. Les parlements de Tours, Chalons et l'assemblée ecclésiastique réunie à Chartres en septembre ne tardèrent pas à s'en prendre aux bulles de Rome : le premier à répondre fut le Parlement de Chalons ${ }^{27}$, ensuite ce fut Henri de Navarre lui-même, par le moyen d'un édit rétablissant les édits de pacification et de lettres patentes ${ }^{28}$.

Ces dernières lettres patentes du Roi portaient,

[la] convocation d'un Saint et libre Concile, par lequel ce qu'il y a de différent et de discordant au fait de la Religion, pût être si bien esclarci et vuidé, qu'il ne pût jamais être en aucune dispute et incertitude [...] ayant cepandant juré et promis que nous ne changerions ou innoverions, ni ne souffririons être rien changé ou innové au fait et exercise de la Religion Catholique, apostolique et romaine, laquelle nous voulons conserver et maintenir [...]

Et aussi sur la défense des libertés de l'Église gallicane, que les bulles et lettres monitoires attaquaient ${ }^{29}$. Le parlement de Tours qui prit le flambeau plus tard élevait la diatribe d'un degré : on pouvait lire dans son arrêt :

A declaré et déclare Gregoire, se disant Pape, quatorziesme de ce nom, ennemi de la paix, de l'union de l'église Catholique, Apostolique, et Romaine, du Roi et de son Estat; adhérant à la conjuration d'Espagne, et fauteur des rebelles; coupable du très cruel, très inhumain et très détestable parricide proditoirement commis en la personne de Henri III, Roi de très heureuse mémoire $[. . .]^{30}$.

Bon dernier, le parlement de Caen rendit un arrêt similaire le 13 août $159^{31}$.

Cette guerre de pamphlets, faite à coups d'arrêts de parlement, continua jusqu'à l'automne avec d'autres ripostes: une du Parlement ligueur de Paris rédigée par Loys Dorléans et le traité de Zampini qui est comme une somme sur cette délicate question et qui clôt la dispute pour l'année $159^{32}$.

Telle était la situation historique de cette année; on doit la garder à l'esprit pour comprendre le "crime» de de Saintes. Deux documents nous aideront à comprendre quelles furent les accusations contre lui. Le premier est un écrit du théologien qui date du mois de mai 1591 et qui était un pamphlet de la propagande ligueuse ${ }^{33}$. Le deuxième est L'histoire anonyme de la Ligue, le texte partiellement édité par Charles Valois en $1914^{34}$. Ce manuscrit, mieux vaudrait dire livre d'histoire sous forme manuscrite, ne parle de de Saintes qu'en 1591. L'auteur se réfère au pamphlet édité avec le nom de l'évêque pour expliquer cette situation particulière. Le récit commence, comme celui des autres historiens avec le 20 janvier de cette année : Biron prit la ville et l'évêque put en sortir pour se retirer à Louviers, ville proche et fortifiée, pendant que Biron nomme $\mathrm{M}$. de Grimonville, sieur de Larchant, gouverneur. Notre auteur rappelle comment de Saintes se souciait de son évêché bien que contraint à l'exil, et comment ses opinions ligueuses ne 
trouvaient pas de sympathie auprès de Larchant et du parlement de Caen où présidait Groulart, « ennemy de l'évêsque ». L'auteur de ce manuscrit écrit :

Or comme c'est la principale charge des Evesques que la predication de la parole de Dieu, pour l'execution de laqu'elle, ils doivent pourvoir des bons predicateurs dans les lieux ou ils ne peuvent eux mesmes prescher, il fut adverty, qu'encores que luy Evesque eust ordonné que le prieur des Jacobins d'Évreux prescheroit la caresme de l'année 1591 en l'esglise cathedrale, Larchant y vouloit faire prescher frere Julian Bernardi.

Julien Bernardi était un jacobin, mais clairement favorable aux idées politiques du parti qui soutenait Henri IV ${ }^{35}$. De Saintes lui écrivit, le 27 février, une lettre dans laquelle, sous peine d'excommunication immédiate, il lui intimait de ne rien entreprendre. De Saintes invoquait l'excommunication pour tous ceux (le parlement de Caen était dans ce cas) qui, contre son autorité et licence, feraient prêcher Bernardi. Il informe d'ailleurs le jacobin qu'il a ordonné au prieur de son couvent de prêcher le carême. Dans cette lettre, de Saintes ajoute qu'autrement, le jacobin Bernardi doit se considérer excommunié selon les canons du Concile de Trente établis contre l'hérésie anglaise pour empêcher que le magistrat commande aux religieux ; le même sort était réservé à tous ceux qui l'auraient imposé comme prédicateur et aussi à d'éventuels auditeurs, qui auraient assisté à son prêche une fois informés de l'excommunication pendante sur la tête de ce jacobin. C'était l'exclusion des sacrements pour les fauteurs et partisans du roi de Navarre. Le même jour, il envoya une lettre au prieur lui ordonnant, toujours sous peine d'excommunication, d'aller prêcher à Évreux, car «vu que la charge lui vient de Dieu et [il] ne doit pas y renoncer par peur des hommes $»^{36}$. L'évêque d'ailleurs lui avait commandé de rendre publique sa lettre.

L'anonyme en relatant ces événements écrit :

Neantmoins au iour de mercredy des cendres qui estoit le 4 de mars, les choses ne se passerent pas avec l'honneur et la decence qui est requise, le peuple voulant obeir a son Evesque et le gouverneur voulant estre obey, cela passa si avant que le parlement seant a Caen

Ce Parlement dans la même occasion donna un arrêt qui ordonnait à deux conseilleurs de se transporter à Évreux, pour s'informer de,

[...] la coniuration, rebellionetseditionet [...] proceder a l'instruction [...] du proces de ceux qui en seront trouvés coupables en faisant inhibition et deffence aux Doyen, chanoines et chappellains, curés, vicaires et predicateurs de reçevoir toute lettre, censures monitions, excommunications ou sentences de de Saintes, et de tous autres, de quelque qualité ou condition qu'ils soient, qui se sont retirés aux villes et places liguées et rebelles au Roy; comme estant nulles et a blasmer et contraires aux constitutions des Sts. canons et decrets, et liberté de l'Esglise gallicane; et pareillement au peuple de leur obeir sur peine d'estre déclarés criminels de leze maiesté, et punis comme perturbateurs du repos publis; et outre a la dite cour ordonné que le dit Bernardi continuera ses sermons predicationsausquellessur pareilles peines que dessus seront tenus d'asister, tant lesdits doyens chanoines et chapelains, principal et regens avec leurs escoliers, que les habitans et bourgeois dela dite ville, en attendant, que plus amplement y ayt esté pourveu par le metropolitain; a enjoint a tous curés, vicaires, et autres gens ayant autorité et puissance d'administrer les sacrements, d'y reçevoir tous les bons et fidels serviteurs du Roy, selon la Ste. observation accoustumée en l'esglise, a peine d'estre pareillement attaints et convaincus du crime de leze Majestés ${ }^{37}$.

Les membres du chapitre de Rouen, ayant pris connaissance de cet arrêt très dur contre l'évêque, donnèrent une sentence le 4 mai par laquelle ils déclarèrent l'excommunication 
décernée par de Saintes contre le religieux Bernardi bonne et valable, condamnèrent l'arrêt du Parlement de Caen comme impie et hérétique, parce qu'il violait la liberté de l'Église,

la hierarchie establie en icelle renversée, la puissance des prelats reiettée, les consciences asservies, et le chemin ouvert pour introduire la tirannie d'Angleterre ;

Tous ceux qui avaient donné cet arrêt, étaient d'ailleurs excommuniés ${ }^{38}$. Aussi le parlement de Rouen se mêla-t-il de l'affaire qui était désormais devenue " politique »; une fois ayant pris connaissance des différentes lettres et pièces du dossier, il donna aussi un arrêt daté du 6 mai 1591 sur la requête présentée par l'évêque de Saintes, pour casser le "prétendu arrest donné par aucun hérétiques, et schismatiques refugiés à Caen, usurpans faussement le nom de cour de Parlement » et il ordonnait la soumission aux ordres de l'évêque :

[...] n'y empescher ledit evesque d'Évreux directement ou indirectement en sa charge episcopale, et ce qui en despend sur peine aux contravenans au present arrest d'estre punis comme criminels de lèze majesté Divine et humaine [...] $]^{39}$.

Après le 6 mai, de Saintes fit publier un libelle où, sous forme de lettre, adressée à ses paroissiens, il rappelait et expliquait les événements ${ }^{40}$.Il rappelait ainsi, en insistant sur les canons de l'Église catholique, qu'il est défendu de donner les sacrements aux hérétiques, sauf, si, au momentde la mort, ils sont pénitents. Donc les partisans de Navarre, fauteurs de l'hérétique et excommuniés par le pape qui seul peut les absoudre, et les gens du parlement de Caen,

veulent introduire en nostre diocese le fondement et commencement de toute heresie, et specialement de celle d'Angleterre avec la tyrannie d'icelle, non seulement sur les biens et corps de nos bons et catholiques diocesains, mais aussi sur leurs ames et consciences, et sur l'exercise de la religion Catholique. Apostolique et Romaine : et faire crime de leze maiesté d'estre catholic, contre et au preiudice de la Saincte Escriture, traditions apostoliques, sainctes constitutions ecclesiastiques, ordonnances des feu tres chrestiens Rois de France jusques à present ${ }^{41}$.

41 De la sorte, qui s'opposerait aux ordres de l'évêque serait considéré coupable de crime de lèse-majesté en se fondant bien évidemment sur l'arrêt du parlement ligueur. Il ajoute :
c'est une part trop grande impudence de dire que tout ce qui procede et vient desdits archeveques ou evesques catholiques est nul, et contraire aux constitutions des sainct canons et decrets, et libertés de l'eglise gallicane. Veu qu'il n'y a rien si conforme aux commande-mens de Dieu, et aux sainctes constitutions ecclesiastiques, que d'obeir aux prelats catho-liques, et qui resistent à l'heretique et ne luy veulent aucunement adherer. Et est faux et iniure trop atroce à l'Église Gallicane de dire que sa liberté gist à ne recevoir les mandemens de leurs prelats, qui font leur devoir de fuir et contredire aux heretiques ${ }^{42}$.

Selon l'auteur de l'Histoire anonyme et aussi selon la lettre de de Saintes, les ordres de l'évêque furent suivis par le peuple d'Évreux au point qu'entre 2000 et 3000 personnes se mettaient en procession tous les jours autour de la cathédrale pour empêcher Bernardi d'y prêcher, lequel d'ailleurs, selon l'anonyme, ne réunissait qu'une vingtaine d'auditeurs. Larchant commanda à ses Suisses d'interrompre ces pratiques manu militari et de forcer la population à assister aux sermons de Bernardi. 


\section{une explication de notre sujet :}

j'ay esté bien adverty que le parlement de Cains procede contre moi pour me degrader du tout, et vous donner un autre evesque; je ne suis pas ignorant de leur mauvaise volonté, et de tous leurs semblables heretiques contre moy, et de leurs inventions, calomnies, et menteries qu'ils ont coustume de faire contre les pasteurs pour prendre le troupeau. Mais ie suis deliberé de ne tenir aucun conte de toutes leurs procedures. Il me suffit de monstrer au doigt qu'ils sont notoirement heretiques, et qu'ils ne peuvent estre mes juges, sans les charger d'avantage [...] quand ils m'auront fait mourir c'est le plus grande honneur et grace que je pourrois recevoir de Dieu en la vieillesse ou je suis [... $]^{43}$.

De Saintes fut donc accusé et trouvé coupable de lèse-majesté selon l'arrêt du parlement de Caen du 28 mars bien avant qu'on découvrit dans ses papiers des textes séditieux. D'ailleurs, l'épître latine contenue dans le Bref advertissement s'en prend expressément à Groulart, président de ce parlement. De Saintes s'était opposé à un parlement qui voulait réglementer les pratiques religieuses et soutenait qu'en vertu de ses fonctions (on pourrait dire au nom de la "Ragione di stato", puisque le livre de Botero est contemporain), et pour la défense de l'Église gallicane, il pouvait, commander aux prêtres d'exécuter ses volontés. De Saintes s'opposa fermement à cette innovation; il est vrai que, pendant les guerres de Religion, la prédication représentait un enjeu politique. Il est évident que les ordres du parlement de Caen étaient inspirés par de très fortes raisons politiques. Mais de Saintes avait-il tort de s'y opposer au nom de la religion? Pour les royalistes bien évidemment oui. Au point qu'ils n'hésitèrent pas à le condamner à mort. Cependant les explications de l'évêque au sujet de l'église gallicane et des saints canons, pour répondre aux accusations du parlement, sont difficiles à contredire du point de l'orthodoxie romaine.

De Saintes se trouva donc en plein milieu d'une guerre menée à coups de bulles, d'excommunications, de lettres patentes et d'arrêts qui aurait pu conduire l'Église gallicane, c'est-à-dire celle que défendaient les parlements royalistes, donc une Église autre que celle dont parlait l'évêque, au schisme. Solution à laquelle on arriva presque en 1592, mais qui, semble-t-il, fut bloquée par la ferme opposition du Cardinal de Bourbon et de du Perron. Mais il s'agit d'une autre histoire.

De Saintes en prison mourut peu de temps après: on soupçonna les «hérétiques » de l'avoir empoisonné, ou bien un neveu ${ }^{44}$; cette affirmation indigne fortement Bayle et lui permet de commencer toute une polémique contre Louis Moreri ${ }^{45}$.

Quoi qu'il en soit, l'anonyme termine ainsi son histoire :

Chacun jugea bien qu'estant renvoyé a Caen pour estre jugé par ceux contre lesquels il avoit escrit cet escrit seroit le plus grans crime dont on le pouroit convaincre ; ainsy mourut cet evesque lequel appellé par ses amis pour se mettre en seureté a Paris ou a Rouen, n'y voulut jamais aller, pour ne pas abandonner son troupeau; la resolution qu'il avoit prise de reçevoir patiemment tout ce que Dieu luy envoiroit, fit qu'il respondit a ses juges avec tant de constance, qu'il en laissa l'estonnement mesme a ses ennemis ${ }^{46}$.

L'auteur de ce texte, contrairement aux autres historiens cités, avait été ligueur et n'avait pas abandonné son credo, mais dans la complexité de son œuvre, écrite au temps de Louis XIII, on peut le définir comme un modéré, tout au moins quand il eut atteint un âge avancé. Dans le cas de l'évêque d'Évreux, bien qu'il place côte à côte les deux versions de l'histoire, il semble cependant défendre la mémoire du fameux théologien avec une 
certaine conviction, qui le fait paraitre, plus qu'un ligueur nostalgique, un fervent défenseur des prérogatives ecclésiastiques ${ }^{47}$.

Les récits royalistes rappellent la vigueur avec laquelle de Saintes défendit ses positions lors de son procès. Cette "arrogance " de l'évêque face à l'accusation d'avoir écrit des textes tyrannicides ou bien régicides est l'exemple pris par de Thou pour prouver la mauvaise foi du prélat et en même temps une condamnation de la théorie ligueuse. Pour l'anonyme, de Saintes défendit ses convictions sur le gouvernement de l'Église. La question est bien donc: quelle idée défendit-il ? Des théories tyrannicides ou bien son attitude pendant la crise du Carême d'Évreux? La reconstruction qui vient d'être faite rend le doute légitime. L'affaire, semble-t-il, fit un grand bruit. Plus pour l'excommunication et la défense de s'approcher des sacrements faits à Bernardi et aux partisans de Navarre que pour la condamnation de l'évêque.

\section{Postérité et nouvelles directions de recherche de I'histoire de la Ligue}

Selon certains récits la Ligue en fit un martyr. Pour les historiens royalistes ou plus tard pour les chroniqueurs du diocèse d'Évreux, il resta un séditieux qui avait reçu ce qu'il méritait ${ }^{48}$. De Saintes bien sûr n'a jamais reçu le statut de martyr que l'église catholique aurait pu lui donner. D'ailleurs, aucun des morts pour la « cause » (l'argument est aussi valable pour Jacques Clément dont après 1594 on n'entendit plus parler), ni aucune des victimes des guerres de Religion en France n'a jamais reçu cette qualification de la part du Saint-Siège. Ce qui est certain est que cette histoire a été en quelque sorte "censurée " par les historiens royalistes ou, en quelque manière, associés au pouvoir d'Henri IV.

On peut conclure que plutôt que de rappeler les vraies causes de la condamnation, les historiens royalistes ont préféré prétexter d'accusations (plus ou moins forgées de toutes pièces) de lèse-majesté et ensevelir dans l'oubli le procès de cet évêque. Seul de Thou rappelle plus longuement l'histoire de l'évêque, mais, comme on a vu, il ne fournit, dans son récit, aucune preuve de l'incrimination ou du chef d'accusation impossible à vérifier, étant donné que les papiers du procès ont disparu. De Thou a tout justifié à travers une formule : la loi du royaume.

Le fait qu'aucun récit du procès n'ait subsisté est déjà en lui-même un élément très important, sinon une preuve. De Saintes, de toute façon était mort, pourquoi rappeler qu'un évêque, un des plus fameux des théologiens engagés contre les protestants, était mort dans une prison d'Henri IV? Loin de vouloir faire de de Saintes un martyr ou d'accuser Groulart et les gens du parlement de Caen d'être des hérétiques, deux points de vue franchement dépassés, à mon sens, comme à celui de l'immense majorité de nos contemporains, le fait plus important de toute cette histoire réside dans l'attitude du parlement de Caen. Au milieu d'une guerre de pamphlets, bulles, et excommunications, ce parlement a eu le couragede choisir qui devait prêcher, de commander aux ecclésiastiques ce qu'ils devaient dire dans leurs sermons pour le Carême, de lever l'excommunication de tous ceux contre qui le chapitre de Rouen l'avait décrétée, et même de condamner un prélat à mort comme coupable de crime de lèse-majesté, en mettant toujours en avant les «libertez de l'Église gallicane». De Saintes, de son côté, par son action prétendait défendre lui aussi les mêmes « libertés de l'église ». On sait que l'évêque a perdu son combat, sa mort en prison en est une démonstration. Mais le simple fait que 
l'on n'ait jamais rappelé dans les récits des historiens royalistes que l'accusation portée contre lui trouvait ses racines dans sa tentative de défendre ses conceptions de la religion et de l'Église contre la mainmise du parlement de Caen constitue peut-être une demivictoire pour le pauvre évêque.

De Saintes, il faut le rappeler, a été condamné pour tout autre crime (ou prétexte) que ceux qui lui avaient été imputés par le parlement de Caen en mars 1591. De cette façon, le parlement (et par la suite les historiographes de Henri IV) en cachant les vraies raisons d'une condamnation prononcée bien avant que de Saintes fût transféré face au tribunal parlementaire de Caen, donne une vision différente des événements. L'accent est mis sur un crime, la possession de livres ou écrits incitant au tyrannicide, dirigé contre la personne du roi Henri IV. On passe donc sous silence le fait que l'évêque avait déjà été condamné comme criminel de lèse majesté pour s'être opposé à la mainmise d'un parlement sur des questions qui concernaient la prédication en temps de Carême.

L'histoire de l'évêque De Sainctes, étudiée dans son contexte politique, montre clairement que les historiens du premier Bourbon ont préféré raconter une réalité différente sinon déformée des événements.

Il se pose donc une question très suggestive pour l'historien d'aujourd'hui qui devrait s'interroger sur l'«histoire " de la Ligue et même la réalité des événements que les historiographes partisans d'Henri IV nous ont légués dans les pages de livres d'histoire célébrés jusqu'à aujourd'hui pour leur objectivité et leur véracité.

\section{NOTES}

1. Denis Pallier, Recherches sur l'imprimerie à Paris pendant la Ligue, Genève, Droz, 1976.

* Désormais, dans le texte, de Saintes.

2. C'est le cas de Pierre Matthieu, historiographe du roy Henri IV, de Palma Cayet, chronographe du même, de Jean-Baptiste Legrain, historiographe de la reine Marie de Médicis et de Jacques Auguste de Thou, l'historien le plus fameux grâce à son Historiae sui temporis, membre du parlement de Paris et chef de file du parti des Politiques, sinon le véritable chef, étant donné que tous les membres du cercle érudit qui se réunissait dans sa maison (la soi-disante « académie de de Thou ») appartenaient à ce parti et avaient pris une position favorable à Henri de Navarre encore protestant.

3. René de Lucinge, La manière de lire l'histoire, éd. critique de Michael John Heath, Genève, Droz, 1993, p. 92-95.

4. Histoire anonyme de la Ligue, BNF, Ms. fr. 23295-23296. Charles Valois en avait donné une édition très partielle en 1914 (Histoire de la Ligue. Cuvre inédite d'un contemporain, Paris, Laurens, 1914). Le texte dans son intégralité est en cours d'édition par les soins de Robert Descimon, MarkGreengrass et Marco Penzi.

5. L'abbé Chemin, Histoire de Claude de Saintes, évêque d'Évreux, publiée par l'abbé Guèry, p.2, Évreux, Impr. de l'Eure, 1892; Frederic J. Baumgartner, Change and Continuity in the French Episcopate, Durham, Duke University Press, 1986, p. 233.

6. L'abbé Chemin, op. cit., p. 3. 
7. L'abbé Chemin soutient que ce livre de 1560 s'intitulait: Commentaire sur les édits des anciens princes touchant la tolérance des sectes dans la religion chrétienne ou méthode que les premiers empereurs catholiques ont gardé contre les sectes (1560), cit., p. 3-4, et 108. Il existe de ce livre une édition latine de 1561, citée aussi par Bayle : De edicta veterum Principum de licentia sectarum in christiana religione. Item methodus contra sectas quam sequuti sunt primi catholici imperatori. Parisiis, Claudius Fremy, 1561 ; Pierre Antoine Bayle : Dictionnaire Historique et critique, t. XIIII, art. Sainctes, p. 28, note B. En français, j'ai pu seulement retrouver l'édition de la deuxième partie de l'ouvrage Discours sur les moyens anciennement pratiqués par les princes catholiques contre les sectes (Paris, Claude Fremy, 1563), qui est également citée par Bayle dans le texte latin.

8. Ibid., Paris, C. Fremy, 1561.

9. Pendant cette période il fera éditer un autre écrit: Discours sur le saccagement des églises catholiques par les hérétiques anciens et nouveaux calvinistes en l'an 1562, Paris, Claude Fremy, 1562, (réed. Paris 1563 et 1567, et toujours à Paris par Jacques Ruelle en 1587). Dans l'édition de 1567 de Saintes ajouta un chapitre sur "l'ancien naturel des français et la religion chrestienne». L'activité de Claude de Saintes au sein de la délégation française au Concile de Trente a été esquissée par Alain Tallon : La France et le Concile de Trente 1518-1563, Rome-Paris, École française de Rome, 1997.

10. Pierre Antoine Bayle : Dictionnaire historique et critique, nouvelle édition, Desoer, Paris, 1820, t.13, Art. Sainctes, p. 28, note B, qui cite Jean de Launoy : Regii Navarrae gymnasii parisiensis historia, Parisiis, apud viduam E. Martini, 1677, p. 778. Launoy relate cette histoire en affirmant que ce fut par le vouloir de Pie V, rendu manifeste par un bref au nonce de Paris, que cette doctrine fut écartée par l'Église Catholique.

11. L'abbé Chemin, op. cit. p. 54-55.

12. Pierre de L'Estoile : Journal de l'Estoile pour le règne d'Henri IV, Paris, Gallimard, 1948, p. 114.

13. Pierre Matthieu: Histoire des derniers troubles de France s.l., s. n., 1606, livre V,p. 31. Il faut signaler que la courte référence à de Saintes est faite dans le récit du siège de Rouen que Matthieu décrit en l'année 1592.

14. Palma Cayet: Chronologie novénaire, Choix de chroniques et mémoires sur l'histoire de France, par J.A.C. Buchon, Paris, Desprez, 1836, vol. I, 1. III, p. 314.

15. Le parlement de Normandie, sous Henri III, après les événements de Blois, s'était divisé en deux : les royalistes siégeaient à Caen, et le Parlement ligueur était resté à Rouen. Le dernier Valois mort, le chapitre de Rouen avait à sa tête le pénitencier Dadré, l'évêque de Lisieux d'Escars, celui d'Évreux Claude de Saintes, celui d'Avranches Péricard, qui combattaient ouvertement Henri IV. Le roi de Navarre avait été reconnu officiellement par le parlement de Caen le 18 août 1589 ; le premier président de ce parlement était Claude Groulart.

16. Cet intertitre, comme tous les autres de l'édition française de l'Histoire de Jacques Auguste de Thou, ne revient pas à l'historien, mais aux éditeurs qui résument ainsi la pensée et le sens que l'auteur a voulu conférer au récit, Jacques Auguste de Thou, Histoire universelle, Londres, 1734, t. XI, l. CI, p. 362.

17. Ibid., t. XI, 1. CI, p. 362-363. L'édition latine définit de Saintes « regis partibus infestissimus ». Historiarum sui temporis, excudi curavit Samuel Buckley, 1733, vol. 5, 1. CI, p. 65.

18. Pierre Antoine Bayle, Dictionnaire historique et critique art. cit., "Sainctes", supra n. 10, p. 29.

19. Jules Lair, Histoire du Parlement de Normandie. Depuis sa translation à Caen au mois de juin 1589, jusqu'à son retour à Rouen, en avril 1594, Caen-Paris, Hardel, 1860, p. 152. Cet auteur raconte aussi une rencontre entre Henri IV et de Saintes: le roi l'aurait pardonné, et l'évêque lui aurait demandé de lui permettre de discuter de théologie, en présence du souverain, avec des protestants, pour lui démontrer qu'elle était la vraie foi.

20. Amable Floquet, Histoire du Parlement de Normandie, Rouen, Emile Frère, 1840-42, t. III., p. 530. L'auteur cite la cote Registres secrets du Parlement ligueur, 23 septembre 1591.

21. Ibid., p. 529-530. Il cite la cote Registres secrets Caen, 10 juin 1591. 
22. Jules Lair, op. cit., note 12.

23. Bref de N. S. Père le pape Grégoire XIIII. envoyé à monseigneur l'évesque de Plaisance. Contenant la première résolution de Sa Saincteté sur les affaires du Royaume de France et de la ville de Paris, Paris, Robert Nivelle et Rolin Thierry, 1591.

24. Traduction du bref de Nostre S. P. le Pape envoyé à Monseigneur le duc de Mayenne, lieutenant général de l'Estat royal et couronne de France, Paris, Jean le Blanc, 1591.

25. Bref de N.S. Père le pape Grégoire XIIII a messieurs de la sacrée faculté de Théologie de Paris : avec la missive de Monseigneur Landriano, nunce de Sa Sainteté en ce Royaume, envoyée ausdits seigneurs, Paris, F. Morel, 1591 ; Bref de N. S. le Pape Grégoire XIIII, a la ville de Paris. Avec la missive de Monseigneur Landriano, Nunce de Sa Sainteté en ce royaume, envoyée à messieurs les prévost des marchans et eschevins de ladicte ville. Paris, F. Morel, 1591.

26. L'une contre toute personnes ecclésiastiques, suyvans le party de Henry de Bourbon, iadis roy de Navarre. L'autre aux princes, seigneurs, nobles et autres personnes laiques suyvans le mesme party, les bulles datent du $1^{\mathrm{er}}$ mars 1591 et on en connaît différentes éditions, parfois séparées, Paris, R. Nivelle et R. Thierry, 1591.

27. Arrest de la cour de Parlement de Châlons. Au mois de juin 1591 dans Mémoires de la Ligue Amsterdam, chez Arkstée \& Merkus, 1758, t. IV, p. 369. On peut y lire: « [la cour de Parlement ...] a cassé, revoqué et annullé, casse, revoque et annulle toutes lesdites bulles, procédures, excommunications et fulminations, comme abusives, scandaleuses, séditieuses, pleines d'impostures, et faites contre les saints décrets, constitutions canoniques, Conciles approuvés, et les droits et libertez de l'Eglise Gallicane. A ordonné et ordonne que si aucuns ont été excommunié par vertu desdits procédures, ils seront absous [...]» Contre le Nonce Landriano on décréta que le «[...] pretendu Nonce, entré clandestinement en ce roiaume, sans congé et permission du roi, sera pris au corps et mené prisonnier ès prisons royales de cette ville de Châlons [...]».

28. Edit du roi contenant rétablissement des Edits de pacification faits par le défunt Roi Henni III, sur les troubles de ce Royaume Chalons 24 juillet 1591. Dans Mémoires de la Ligue, op. cit., t. IV, p. 358-361

29. Lettres patentes du roi, contenant déclaration de l'intention qu'il a pour maintenir l'eglise et Religion Catholique, Apostolique et Romaine en ce Royaume; ensemble les droits et anciennes libertez de l'eglise Gallicane dans Mémoires de la ligue, op. cit., t. IV, p. 361-367.

30. Arrest de la cour de Parlement seante à Tours. sur les Bulles Monitoriales de Grégoire, se disant pape [ ...] dans Mémoires de la Ligue, t. IV, p. 367-369.

31. Intéressant est le réquisitoire de l'avocat du roi Thomas de Verdun : rappelant à ses collègues leurs prédécesseurs et leur attitude en pareils cas, il soutint que: «L'Église gallicane est exempte de toutes prétendues excommunication et interdictz. Les papes mesmes les plus ardents et audacieux ont esté contrainctz le confesser par plusieurs bulles authentiques. En cas de commination ou déclarations de telles censures, on s'y est toujours unanimement opposé, tant pour soustraction d'obeissance que de pécune et finances. » Grégoire XIV avant de publier de telles bulles «libelles diffamatoires, scandaleus [...] par son pretendu Nonce » aurait dû s'en souvenir. Il demandait ensuite: « appellant comme d'abus du pretendu rescript ou bulle en forme d'excommunication et interdict; que Marsilius Landrianus, prétendu nonce, fût , comme ennemy public et boutefeu, couru et poursuivy, pris vif ou mort [...]». Amable Floquet, Histoire du Parlement de Normandie, op. cit., t. III, p. 534-535.

32. Arrest de la cour de Parlement contre certain pretendu arrest donné à Tours sur le faict des bulles monitoires de Nostre Sainct Père. Paris, Robert Nivelle, 1591 ; Matteo Zampini: Ad Calumnias, et imposturas, a pseudo-parlamentis Cathalaunensi et Turonensi ac Carnotensi conventiculo, ad catholicae religionis perniciem populique deceptionem impie conflictas in Gregorium XIIIII illiusque monitionis literas, ad clerum, principes, nobiles et populos Franciae, responsio Matthaei Zampini Recanatensis, I. C., Paris, Rolin Thierry, 1591. 
33. Bref advertissement de monsieur l'évesque d'Évreux à ses diocésains contre un prétendu arrest donné à Caen, le 28 de mars dernier, par lequel il appert de l'introduction et establissement en France du schisme, hérésie et tyrannie d'Angleterre. avec ledit arrest, sentence du métropolitain et arrest de la Cour donnez contre iceluy. Suivant la copie imprimée à Rouen,Paris, Guillaume Bichon, 1591.

34. Histoire de la Ligue: œuvre inédite d'un contemporain, Paris, H. Laurens, 1914. Les références données ici sont tirées du manuscrit de la BNF, Ms fr 23296, que Valois n'avait pas édité.

35. BNF, Ms. fr. 23296, p. 416.

36. ibid., p. 417.

37. BNF, Ms. fr. 23296, op. cit., p. 417- 418.

38. Ibid., p. 418.

39. Ibid., p. 419.

40. Bref advertissemnt de Monseigneur [...], op.cit., il contient au $\mathrm{V}^{\mathrm{e}}$ titre «Ad inclytam Rothomagnum ", l'épître latine adressée à Claude Groulart signée «Ph. Hispanus I.V.C. » (Philippe évêque de Plaisance); l'advertissement est suivi, p.17, des lettres de Claude de Sainctes à Bernardi, Jacobin, et à son prieur, datées de Louviers, 24 février 1591. Il contient à la p. 21, l'arrêt du «prétendu parlement de Caen » du 28 mars; à la p. 24 "Sententia capituli Rothomagensis » du 4 mai ; et à la p. 28, arrêt du parlement de Rouen contre l'arrêt précédent, du 6 mai 1591.

41. Bref advertissement [...], op. cit., p. 4

42. Bref advertissement [...], op. cit., p. 7-8 « Cet arrêt [dit l'évêque] porte des hérésies et tyrannies plus grandes que celles d'Angleterre ou du Turc, au point que le Parlement devrait se nommer de Caïn plutôt que de Caen ». Dans ce texte, de Saintes explique mieux quelle est la théorie de l'Église Catholique Apostolique et Romaine et donc sa pensée : "C'est heresie et commencement de toute heresie, de iuger estre loisible à homme que ce soit de prescher en la chaire ou esglise d'un evesque catholique, non seulement sans la licence et permission des superieiurs de l'eglise, mais nonobstant leur deffence et excommunication signifiée à celui qui le voudroit entreprendre. C'est heresie de iuger qu'il soit loysible à aucun que ce soit, de prescher en une esglise d'aucun diocese par authorité, force et violence du magistrat seculier, nonobstant la deffence et excommunication donnee contre celuy qui l'entraprendera par les superiuers de l'eglise catholique. C'est heresie de iuger estre licite à un magistrat seculier de disposer de la religion et predication catholique, et d'y commetre qui bon luy semblera, sans la licence des superieurs (p.11) de l'eglise catholique ou mesmes contre leur deffence et excommunication » (p. 12). C'est heresie de commander à un du clergé excommunié de prescher contre la volonté des superieurs et de contraindre le peuple à l'écouter sous peine de leze majesté. C'est heresie de iuger estre loisible à un curé de faire parteciper aux sacrements les heretiques excommunié par la bulle en Coena Domini que par les excommunication particulieres du pape. C'est encore plus grande heresie de iuger estre licite commander aux curez de ce faire, sur peine de crime de leze maiesté » (p. 13). Il ajoute que c'est aussi hérésie de priver les évêques et autres prélats de leur puissance s'ils ne veulent pas adhérer à un roi hérétique et d'affirmer qu'il est illicite de ne pas adhérer à un roi hérétique, comme chose contraire aux saints canons, et aussi d'ordonner au peuple de n'obéir aux ordres, lettres etc. de leurs évêques ; ces ordres qui peuvent légitimement demander au peuple de se retirer des villes de l'ennemi pour ne pas lui obéir. Il affirme que c'est aussi hérésie de juger qu'un magistrat séculier a le pouvoir de destituer un évêque (p. 10-11). En outre, il en appelait au Cardinal de Bourbon, le même métropolitain nommé dans l'arrêt du parlement de Caen, qui aurait dû nommer un suppléant à de Saintes, et le suppliait d'éviter qu'une telle injustice soit commise.

43. Bref advertissement [...], op. cit, p. 16-17.

44. «Ad castrum ergo de Crepicordio diocesis Lexoviensis ablegatus, et in ergastulum conjectus, ibidem non sine suspicione veneni ab haeretis, vel ut quidam volunt, a nepote, quae ex perturbatione rerum deteriorum aliquid reformidabat, propinati, alias ultima necessitate, decessit anno 1591, aetatis 65 », Gallia Christiana, t. XI, col. 612-613. 
45. Louis Moréri, Le grand dictionnaire historique, ou mélange de l'histoire sacrée et prophane, Lyon, J. Gyrin et B. Rivière, 1681. "Quelle absurdité que de prétendre qu'ils l'aient empoisonné ? Il ne pouvait plus leur nuire ; car encore qu'il eût echappé par grâce à la main du bourreau, il devait vivre tout le reste de ses jours dans une prison.» (p. 32 art. cit., "Sainctes »). L'idée d'empoisonnement dut circuler tôt, l'auteur de l'Histoire anonyme, rédigée vers 1620, dit à ce sujet: « ou il mourut deux jours apres, non sans soupçon qu'on avoit avancé la mort de ce grand prélat », op. cit., p. 423.

46. Ibid.

47. Frederic J.Baumgartner note : «Except for the executes cardinal de Guise, de Saintes came the closest to being a casualty of the conflict over the royal succession that the episcopate suffered from 1585 to 1595 . That fact is quite remarkable in view of the tremendous range of political and military activities in which the bishops were involved for the two parties ", op. cit., p. 171. Cette affirmation met en évidence la sévérité du jugement qu'il subit.

48. Pour la vision du martyr, cf. le récit de l'Histoire anonyme de la Ligue, cité, et Gallia Christiana t. XI col. 612-613; Histoire civile et ecclésiastique du comté d'Évreux, Paris, F. Barois, 1722, p. 362, qui soutient qu'il fut ainsi considéré par les Ligueurs, bien que le texte soit très sévère contre l'engagement politique de de Saintes. Pour la version royaliste, mis à part de Thou, cf. Bayle, art cité ; Amable Floquet, cité, donne un jugement très négatif sur l'attitude de l'évêque. Victor de Chalambert, Histoire de la Ligue sous le règne de Henri III et Henri IV, ou quinze années de l'histoire de France, Genève, Slatkine-Megariotis reprints, 1974, a une vision modérée : il «fut condamné à une réclusion perpétuelle ; c'était un prélat plein de science et de piété, connu pour son attachement à la cause de l'Union. Il mourut peu de temps après dans sa prison » (p. 252).

\section{AUTEUR}

MARCO PENZI

EHESS/CRH 